\title{
Correspondence
}

\section{POGO Score}

To the Editor:

The results of Ochroch and colleagues' comparing percentage of glottic opening (POGO) score vs Cormack and Lehane grading. ${ }^{1}$ confirm previous work by Cohen and colleagues, who showed that trained anesthesiologists are frequently unable to assign Cormack and Lehane grading correctly to a picture of laryngeal view. ${ }^{2}$ Less than half of 120 British anesthetists correctly graded a picture of a difficult, grade 2 view.

Ochroch's study clearly identifies that greater sensitivity can be achieved using their POGO score. However providing some part of the glottis can be seen intubation difficulty is rare. The majority of difficult intubations occur when no part of the glottis can be seen. Rose studying more than 3000 patients found $84 \%$ of difficult intubations and all failed intubations were associated with inability to see the vocal cords. ${ }^{3}$ These cases are Cormack and Lehane grade 3 -4: POGO score will always be $0 \%$ and so will provide no discriminating information.

I have described ${ }^{4}$ and recently evaluated a new practical classification of laryngeal view. ${ }^{5}$ The laryngeal view is easy (E) if any part of the glottis is visible. It is restricted ( $\mathrm{R}$ ) if only the posterior glottic structures (arytenoids, posterior commisure) are visible or when only the epiglottis is visible and it can be lifted. It is difficult (D) if only the epiglottis is visible but it cannot be lifted or if no glottic structures can be seen. Studying 500 cases the ERD classification correlated significantly better than Cormack and Lehane's grading with increasing difficulty with intubation (time taken and intubation aids needed).

T.M. Cook MBBS BA FRCA

Bath, UK

References

1 Ochroch EA, Hollander JE, Kush S, Shofer FS, Levitan $R M$. Assessment of laryngeal view: percentage of glottic opening score $v s$ Cormack and Lehane grading. Can J Anesth 1999; 46: 987-90.

2 Rose DK, Cohen MM. The incidence of airway problems depends on the definition used. Can J Anaesth 1996; 43: 30-4.
3 Cohen AM, Fleming BG, Wace JR. Grading of direct laryngoscopy. A survey of current practice. Anaesthesia 1994; 49: 522-5.

4 Cook TM, Tuckey JP. A comparison between the Macintosh and the McCoy laryngoscope blades. Anaesthesia 1996; 51: 977-80.

5 Cook TM. A new practical classification of laryngeal view. Anaesthesia 1999; (in press).

REPLY:

Thank you for your letter. ${ }^{1}$ Our research and that of Rose and Cohen, shows that Cormack and Lehane grading has poor inter-observer reliability for assessing laryngeal exposure. ${ }^{2}$ The POGO score is about quantification of laryngeal exposure. It does not address the prediction of difficult intubation, nor the difficulty of passing a tracheal tube. Adnet et al. has proposed an intubation difficulty scale that addresses seven components of intubation difficulty, only one of which is laryngeal exposure. ${ }^{3}$ In his study, many of the more difficult intubations (higher IDS scores) occurred despite adequate laryngeal exposure, while many of the intubations with lower IDS scores occurred in the setting of CL grade 3 views. For this reason, we believe it is best to separate laryngeal exposure and intubation difficulty. Although your scale has a simplistic descriptive appeal, it confuses the two issues by using the terms "easy" and "difficult" to describe different amounts of laryngeal exposure. It appears as though you are really trying to characterize intubation difficulty.

We believe that percentage of glottic opening score is a useful research tool for quantifying laryngeal exposure, particularly when used in conjunction with videotaping of laryngeal view. ${ }^{4}$ This research methodology can be used to assess exposure with different blades, the effect of laryngeal manipulation, and other aspects of direct laryngoscopy. For describing intubation difficulty, we suggest that Adnet et al. have appropriately included many other variables, in addition to laryngeal exposure, that impact on intubation difficulty. ${ }^{5}$

Richard M. Levitan MD

E. Andrew Ochroch MD

Judd Hollander MD

Philadelphia, USA 


\section{References}

1 Ochroch AE, Kush S, Stuart S, Hollander JE, Levitan $R M$. Assessment of laryngeal view in direct laryngoscopy: the percentage of glottic opening (POGO) score compared to Cormack and Lehane grading. Can J Anesth 1999; 46: 987-90.

2 Rose DK, Cohen MM. The incidence of airway problems depends on the definition used. Can J Anaesth 1996; 43: 30-4.

3 Adnet F, Borron SW, Racine SX, et al. The intubation difficulty scale (IDS): proposal and evaluation of a new score characterizing the complexity of endotracheal intubation. Anesthesiology 1997; 87: 1290-7.

4 Levitan RM. Direct laryngoscopy imaging: teaching and research applications. Educational Synopses in Anesthesiology and Critical Care Medicine (June, 1998): The On-line Anesthesia Journal, http://gasnet.med.yale.edu/esia/1998/june/samart.html. Reprinted: American Journal of Anesthesiology 1999; 26: 39-42.

5 Benumof JL. Intubation difficulty scale: anticipated best use (Editorial). Anesthesiology 1997; 87: 1273-4.

\section{Transesophageal echocardiography in the management of anaphylactic shock}

To the Editor:

We would like to present the use of transesophageal echocardiography (TEE) in the management of anaphylactic shock.

After infusion of dextran, a 47-yr-old man suffered an anaphylactic reaction, consisting of hypotension and bronchospasm. Prednisolone and aminophylline were given and, after midazolam and rocuronium, the trachea was intubated. Large amounts of pink frothy fluid appeared in the endotracheal tube. In the ICU, mechanical ventilation was started with PEEP of $16 \mathrm{~cm}$ $\mathrm{H}_{2} \mathrm{O}$. A pulmonary artery catheter was inserted and a CVP of $20 \mathrm{mmHg}$ and pulmonary capillary wedge pressure (PCWP) of $26 \mathrm{mmHg}$ were measured. Chest $\mathrm{X}$-ray showed massive pulmonary edema. Continuous infusion of epinephrine was started to maintain blood pressure (BP), but without success.

A TEE examination showed good myocardial contractility and no cardiac tamponade. Ventricular dimensions were small. However, CVP and PCWP were elevated, indicating a small circulating blood volume in a contracted vascular system, caused by massive extravascular fluid trapping in the lungs and vasopressor-induced vasoconstriction. Under TEE monitoring,
$1500 \mathrm{ml}$ of a gelatin colloid were infused until the ventricular dimensions appeared normal. The $\mathrm{BP}$ and $\mathrm{SaO}_{2}$ improved to normal, while PAP and PCWP remained unchanged. Epinephrine was discontinued and hemodynamic variables remained stable.

Although infusion of fluids is generally necessary to treat hypotension during anaphylactic shock, we felt this may be hazardous in the presence of high PCWP and pulmonary edema. TEE was helpful in evaluating the intravascular volume status, and complemented pressure measurements of the PA catheter.

W. Anton Visser

Frederik W. Santman

Helmut R. Gehlmann

Wim R.M. Aengevaeren

Nijmegen, The Netherlands

\section{Blind intubation using the Endotrol tube and a light wand}

To the Editor:

The Endotrol tracheal tube (Mallinckrodt, Athlone, Ireland) is useful for blind nasotracheal intubation, since the curve of the tube can be controlled by a wire hook. ${ }^{1,2}$ We suggest that the use of a light wand (Trachlight [Laerdal Medical, New York, USA]) adds to the usefulness of the Endotrol tube.

A 25-yr-old woman had a fractured jaw a few months ago and was scheduled for reconstruction of the jaw. Preoperatively, the mouth opening was restricted to two-fingers width. General anesthesia was induced, and after adequate ventilation was confirmed vecuronium was injected. An Endotrol tube was inserted into the nose and oral laryngoscopy was attempted. Since the glottis could not be seen, blind tracheal intubation was attempted. However, location of the glottis was difficult due to the lack of spontaneous breathing, and intubation failed after three attempts.

Trachlight-aided blind nasotracheal intubation was then attempted while a fibreoptic bronchoscope was being prepared. After the inner metal stylet has been removed from the Trachlight, the wand was inserted into the Endotrol tube. By advancing the tube, illumination was seen in the side of the neck. This time, the tube position could easily be adjusted to the centre by rotating the tube and pulling the hook. The tube was further advanced, the wand was removed and the correct position was confirmed. The time for tracheal intubation was less than $30 \mathrm{sec}$. 